\title{
Automated Flow Injection Techniques in Pharmaceutical Analysis: A Useful tool
}

\section{Paraskevas D. Tzanavaras*}

Laboratory of Analytical Chemistry, Department of Chemistry, Aristotelian University of Thessaloniki, GR-54124 Thessaloniki, Greece

Automation is a critical demand in modern pharmaceutical analysis and quality control, since strict legislation regarding Good Laboratory (GLP) and Manufacturing Practice (GMP) require extensive analyses of huge amounts of samples during all stages of the manufacturing process of a pharmaceutical formulation. The scope of this editorial is to highlight the usefulness of automated flow-injection based analytical techniques in the field of pharmaceuticals quality control. Particular attention will be paid to chemiluminescence detection, flow optosensors and the relatively novel concept of Sequential Injection Chromatography (SIC).

Flow injection based automated analytical techniques include a well-established group of techniques with numerous widespread applications in quantitative chemical analysis. The most popular representatives are Flow (FI) and Sequential Injection Analysis (SI). In contrast to conventional batch and continuous flow procedures, FI and SI do not rely on complete mixing of sample and reagent(s) (physical homogenization). Combined with the inherent exact timing of all events it is neither necessary to wait until all chemical reactions have proceeded to equilibrium (chemical homogenization). These features, that allow transient signals to be used as the readout, do not only permit the procedures to be accomplished within a very short time (typically in less than $30 \mathrm{~s}$ ), but have open new and novel avenues to perform an array of chemical analytical assays, which are either very difficult and in many cases directly impossible to implement by traditional means [1]. Representative FI and SI manifolds can be found in Figures 1 and 2 respectively, while the interested reader can find many articles describing the principles of operation [2-4]. Among the numerous automation potentials of these techniques in pharmaceutical analysis some particularly interesting features including: (i) derivatization reactions; (ii) on-line dilution; (iii) on-line solid phase extraction; (iv) On-line solvent extraction.

Chemiluminescence (CL) is a very attractive and popular technique when it comes to pharmaceutical analysis. FI has given a real boost to the investigation, development and application of novel chemiluminescence reaction schemes. The majority of these reactions are extremely rapid and their adaptation under batch conditions is impractical. However, based on the precise handling and timing of FI,

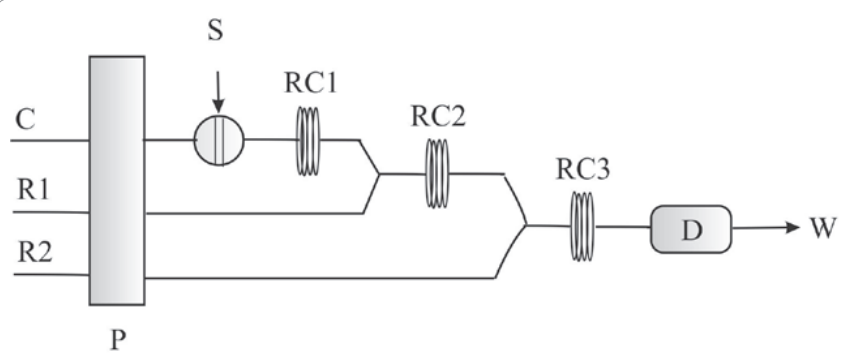

Figure 1: Typical three-streams FI manifold: $\mathrm{C}=$ carrier; $\mathrm{S}=$ sample; $\mathrm{R} 1, \mathrm{R} 2$ = reagents; $\mathrm{RC} 1, \mathrm{RC} 2$, and $\mathrm{RC} 3$ = reaction coils; $\mathrm{P}=$ pump; $\mathrm{D}=$ detector and $\mathrm{W}=$ waste.
FI-CL has evolved to a very "happy marriage" [5]. Available modes may include the determination of either enhancers or inhibitors of chemiluminescence-based reactions. From an instrumentation point of view flow-through chemiluminescence detectors are cost-effective and can be even constructed in-house. Conventional HPLC fluorescence detectors can often be used as alternatives by simply turning off the excitation source. When it comes to analytical figures of merit such systems are generally characterized by low detection limits and wide determination ranges. The main drawback that may be mentioned and should be given particular attention in new methods is the limited selectivity that can be eliminated by coupling CL detection as a postcolumn derivatization system in HPLC.

FI optical sensors are based on the "Solid Phase Spectrophotometry" (SPS) technique which was originally reported by Yoshimura et. al. [6] and has been applied ever since to various types of analytes. Automated FI-SPS sensors involve the use of a suitable solid support (typically silica gel or cation / anion exchange resins) packed in the flow-cell of an optical detector. Analyzed samples are injected in the carrier stream of the FI system and driven towards the detector where the analyte is retained and detected on the solid support. The key features of this approach is that all necessary steps including a) retention of the analyte and separation from sample matrix, b) preconcentration of the analyte and c) detection proceed simultaneously. The analysis cycle is completed by quantitative elution of the retained species and regeneration of the packing material by either the carrier itself or a suitable eluent. A very interesting group of methods based on the use of FI optical sensors involves simultaneous multi-analyte determinations. These methods take advantage of the differences in the retention behavior of the analytes on separation mini-columns located before the sensing zone. Applications of FI-based optical sensors in pharmaceutical analysis can be found in $[7,8]$.

One of the most recent additions to the "family" of automated flow-injection techniques with significant impact in pharmaceutical analysis is the concept of Sequential Injection Chromatography (SIC) [9]. SIC is based on the incorporation of short monolithic stationary phases in the low pressure flow manifold. The pressure drop across 5-50 $\mathrm{mm}$ long commercially available monolithic columns is compatible to most SI setups. SIC enables the reversed phase separation of mixtures

*Corresponding author: Paraskevas D. Tzanavaras, Laboratory of Analytical Chemistry, Department of Chemistry, Aristotelian University of Thessaloniki, GR-54124 Thessaloniki, Greece, Tel: 0030 2310997721; E-mail: ptzanava@ chem.auth.gr

Received July 09, 2011; Accepted July 09, 2011; Published August 03, 2011

Citation: Tzanavaras PD (2011) Automated Flow Injection Techniques in Pharmaceutical Analysis: A Useful tool. Pharm Anal Acta 2:105e. doi:10.4172/21532435.1000105e

Copyright: (C) 2011 Tzanavaras PD. This is an open-access article distributed under the terms of the Creative Commons Attribution License, which permits unrestricted use, distribution, and reproduction in any medium, provided the original author and source are credited. 
Citation: Tzanavaras PD (2011) Automated Flow Injection Techniques in Pharmaceutical Analysis: A Useful tool. Pharm Anal Acta 2:105e. doi:10.4172/2153-2435.1000105e

Page 2 of 4
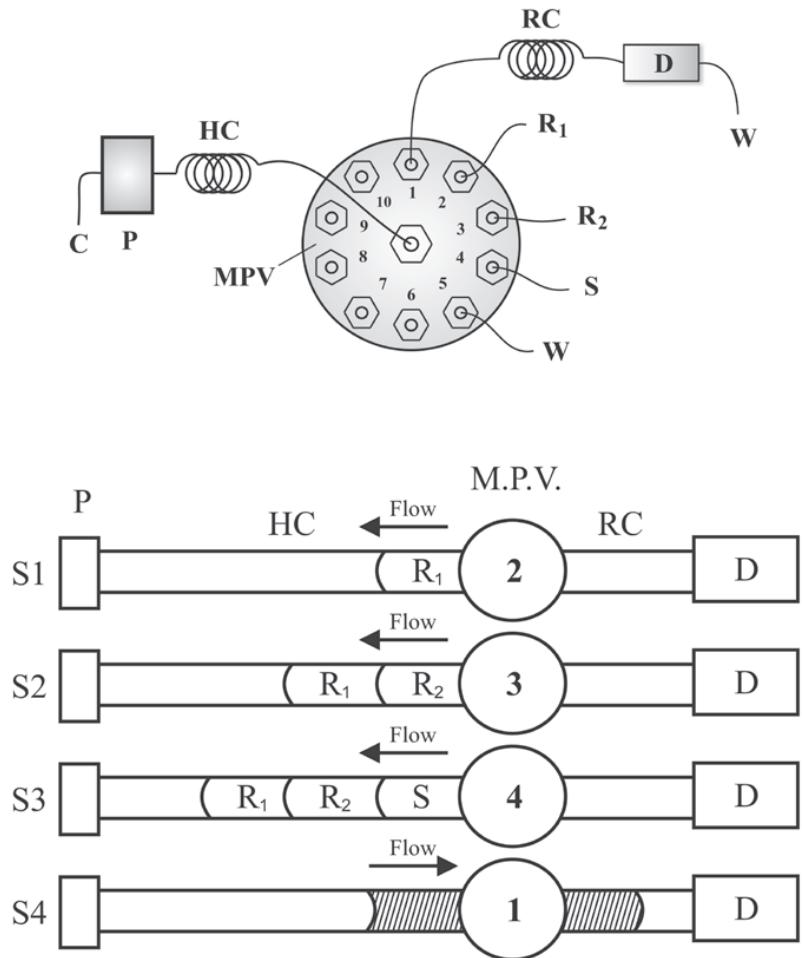

Figure 2: Typical $\mathrm{SI}$ setup and $\mathrm{SI}$ sequence steps: $\mathrm{P}=$ pump; $\mathrm{C}=$ carrier solution; $\mathrm{HC}=$ holding coil; $\mathrm{MPV}=$ multi-position valve; $\mathrm{RC}=$ reaction coil; $\mathrm{D}=$ detector; $\mathrm{W}=$ waste; $\mathrm{R}_{1} \& \mathrm{R}_{2}=$ reagents; $\mathrm{S}$ = sample; $\mathrm{S} 1-\mathrm{S} 4$ = SI steps.

of analytes expanding the possibilities of SI from simple assays to even purity profiling of pharmaceutical formulations [10], while both isocratic and gradient modes are feasible [11]. Recent review articles have highlighted the progress, theoretical background and practical applications of this novel technique [12,13].

Concluding this short editorial piece, my opinion is that flow-based automated techniques can play a viable role in pharmaceutical analysis.
Future work should emphasize on the development of robust and fully validated methods that can prove reliable under industrial testing and "survive" inspection from international authorities. Simplicity is a key demand, while someone could even take advantage of the reliability of commercially available HPLC instrumentation.

\section{References}

1. Ruzicka J, Hansen EH (1981) Flow Injection Analysis. John Wiley and Sons Tzanavaras PD (2011) Automated determination of captopril by flow and sequential injection analysis: A review. Anal Lett 44: 560-576.

2. Economou A, Tzanavaras PD, Themelis DG (2007) Sequential-injection analysis: A useful tool for clinical and biochemical analysis. Curr Pharm Anal 3: 249-261.

3. Economou A (2005) Sequential-injection analysis (SIA): A useful tool for on-line sample-handling and pre-treatment. TrAC - Trends Anal Chem 24: 416-425.

4. Mervartová K, Polášek M, Martínez Calatayud J (2007) Recent applications of flow-injection and sequential-injection analysis techniques to chemiluminescence determination of pharmaceuticals. J Pharm Biomed Anal 45: 367-381.

5. Yoshimura K, Waki H, Ohashi S (1976) lon-exchanger colorimetry-I. micro determination of chromium, iron, copper and cobalt in water. Talanta 23: 449454.

6. Tzanavaras PD, Themelis DG (2007) Review of recent applications of flow injection spectrophotometry to pharmaceutical analysis. Anal Chim Acta 588: $1-9$

7. Rocha FRP, Raimundo Jr. IM, Teixeira LSG (2011) Direct solid-phase optical measurements in flow systems: A review. Anal Lett 44: 528-559.

8. Huclová J, Šatínský D, Karlíček R (2003) Coupling of monolithic columns with sequential injection technique: A new separation approach in flow methods. Anal Chim Acta 494: 133-140.

9. Zacharis CK, Verdoukas A, Tzanavaras PD, Themelis DG (2009) Automated sample preparation coupled to sequential injection chromatography: On-line filtration and dilution protocols prior to separation. J Pharm Biomed Anal 49: 726-732.

10. Koblová $P$, Sklenářová $H$, Chocholouš $P$, Polášek $M$, Solich $P$ (2011) Simple automated generation of gradient elution conditions in sequential injection chromatography using monolithic column. Talanta 84: 1273-1277.

11. Kika FS (2009) Low pressure separations using automated flow and sequential injection analysis coupled to monolithic columns. J Chromatogr Sci 47: 648655

12. Chocholouš $P$, Solich $P$, Šatínský D (2007) An overview of sequential injection chromatography. Anal Chim Acta 600: 129-135. 